\title{
An evaluation of public truck terminal implementation and truck ban policy in a medium-size city of Thailand: a case study of Khon Kaen city
}

\author{
Vuchlung Chhorn ${ }^{1}$, Jaruwit Prabnasak ${ }^{1 *}$, and Chow Chompoo-Inwai ${ }^{2}$ \\ ${ }^{1}$ International College, King Mongkut's Institute of Technology Ladkrabang, Bangkok, Thailand \\ ${ }^{2}$ Faculty of Engineering, King Mongkut's Institute of Technology Ladkrabang, Bangkok, Thailand
}

\begin{abstract}
This study aims at investigation the potential impacts of a public truck terminal implementation along with truck ban policy adaptation in Khon Kaen City, Thailand. A four-step model is developed and used for examining traffic condition changes due to the terminal relocation. Three different terminal locations are set. Truck traffic of each scenario are estimated and converted to total network cost, and then compared with the base-case scenario. The most suitable truck terminal location is suggested. Also, several concerns regarding the public truck terminal and truck ban policy in the study area are given.
\end{abstract}

\section{Introduction}

In large urban area, truck traffic is known as one of the major concern and one popular scheme to tackle is "truck ban policy". Although truck ban policy is well accepted by a large number of cities, banning large vehicles can reduce problems, such as parking and loading issue, traffic obstruction, vibration, air and noise pollution and road accident. However, some studies have been found that the policy can cause increase in direct cost to the truck operators $[1,2]$.

In 2015, the Department of Land Transport proposed a project to develop a number of truck terminals in major regional cities in the country [3]. This leads to a research question that: what would potentially be the result if an urban truck terminal is implemented along with truck ban policy. Therefore, this study aims at investigating the impact of truck terminal implementation along with truck ban policy using Khon Kaen as the principal study area.

\section{Methodology}

The four-step modeling technique [4] is employed. Data used for developing the model was collected in Khon Kaen in 2015. Three different truck terminal location scenarios are set in the study. The total of vehiclekilometer-travelled (VKT) and vehicle-hour-travelled (VHT) of each scenario is calculated by the model on the daily basis. The vehicle-operating-cost (VOC) used in the study is referred to [5].

Fig. 1 presents the assumption of the truck terminal locations for the purpose of examining the impact of truck ban policy. Three test locations are defined by land-use, town master plan, and referred to a study on truck terminal development held by the Department of Land Transport recently [3].

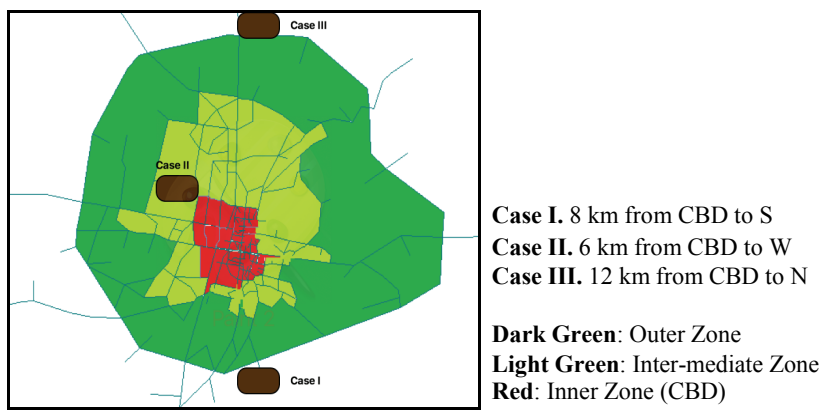

Fig. 1. Three truck terminal location scenarios

\section{Model Results and Discussion}

This section presents model estimated results and discussions for Base-Case and the three test scenarios.

\subsection{Actual Truck Traffic Behaviors in the Study Area (Base-Case Scenario)}

In this section, Base-Case scenario is developed in order to reveal the actual truck traffic behavior currently in the study area. The estimated result was shown in Table 1 .

Table 1. Model estimation results for truck traffic behaviors in base case scenario

\begin{tabular}{|l|c|c|}
\hline Daily truck demand (24 hours) & 3,521 & PCU \\
\hline Total daily truck travel distance & 58,504 & P-kilometer \\
\hline Total daily truck travel time & $1,020.08$ & PCU-hour \\
\hline
\end{tabular}


Table 1 reveals that the total demands of trucks for the normal operation with large trucks were approximately 3,521 PCU (about 16,671 tons of light trucks) moving inside city area in the road networks for 24 hours. In addition, it consisted of 58,504 vehicle-kilometers within the networks. This results in accounting for 1,020 hours with the average trip length of 0.7 kilometers as well as the average trip time around 0.81 hour with average trip speech $49.1 \mathrm{~km} /$ hour.

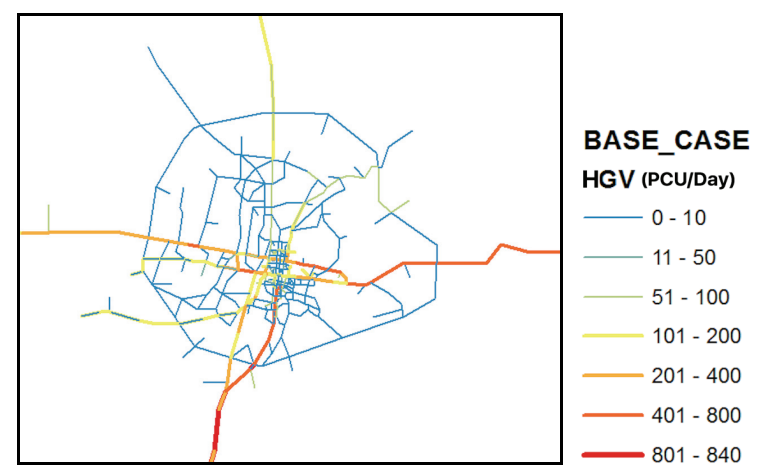

Fig. 2. HGV traffic flow in base-case scenario

In Fig. 2 demonstrates the truck traffic flow on the links for Base-Case scenario. It is showed that the traffic on the link arrives from all direction of the street within the study area. All trucks directly go inside the city area which lead to the heavy congestion problem on the link road as can be seen the yellow line and read line. The red line indicates the heavy traffic on the road. This result makes this assumption becoming more realizable due to the movement of traffic from everywhere crossing city center or coming from city center.

\subsection{Case I Scenario}

For Case I, the truck terminal is located 8 kilometers to the South of the CBD. In Table 2, it is found that the truck demands moving in outer zone are about 3,521 PCU which approximately around 8,802 Ton for large trucks which is moving outside to truck terminal Zone 71 and the truck demands which need to move inside city is about $8,337 \mathrm{PCU}$ which roughly converted to 16,674 tons. This was double differences of the existing demands with the total vehicle hours around 2,152 hours with the same study area.

Table 2. Model estimated result of Case I

\begin{tabular}{|l|c|c|}
\hline Daily truck demand HGV & 3,521 & PCU \\
\hline Daily truck demand DGV & 8,337 & PCU \\
\hline Total daily truck travel distance & 118,843 & P-kilometer \\
\hline Total daily truck travel time & $2,152.60$ & PCU-hour \\
\hline
\end{tabular}

Fig. 3 presents that, after having a truck terminal in the Case I location, the HGVs (Heavy Goods Vehicles) switch to use the outer ring road instead of inner city roads. Then all large trucks that move from outside city turning to use outer link road as shown as the yellow line and orange line through circle around map.

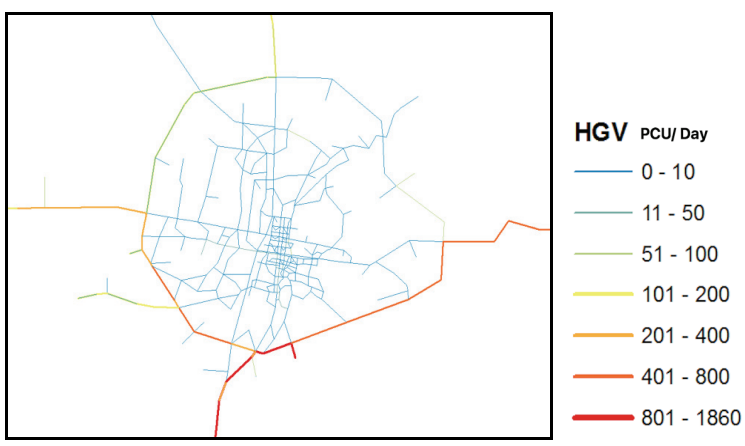

Fig. 3. HGV Traffic flow on links in Case I

Meanwhile, Fig. 4 indicates that the traffic flows on link road within CBD area with the DGV (Delivery Goods Vehicles - are assumed to be 2-Ton Pickup Trucks) moving within $24 \mathrm{hrs}$ operation. The result shows that traffic moving desire line is as per expected with 8,337 PCU (about 16,674 tons). The red color indicates the traffic along the small block from intermediate zone. The traffic is partly crowded until the truck terminal zone. However, as the truck movements are already converted from larger trucks to the small trucks, this is assumed that the traffic flow on the link is normal with small demand of large trucks on link.

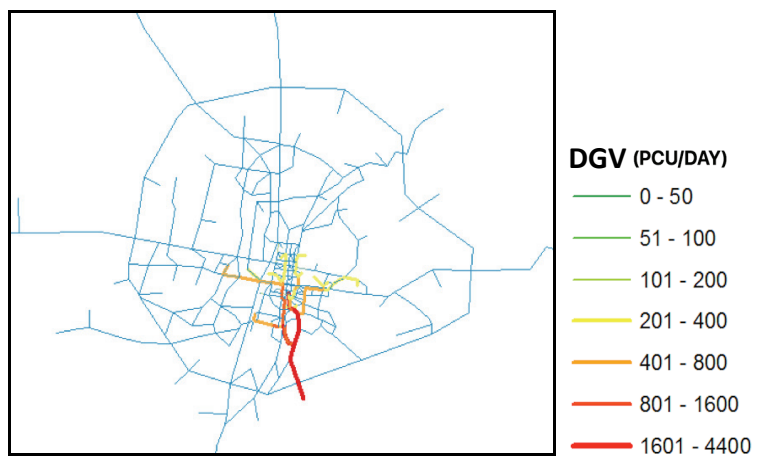

Fig. 4. Delivery Traffic flow on links in Case I

\subsection{Case II Scenario}

For Case II, the truck terminal is assumed to be located 6 kilometers to the West of the CBD (Inside the ring road). Results in Table 3 shows the same demand of HGV and DGV as Case I, however the differences are found between total truck traveling distances about 290,939 PCU-Kilometers which was 144 percent greater than Case I and truck traveling time about 4,841 PCUhours which is about 125 percent greater than Case I. This obviously shows that Case II are not satisfied the study expectation.

Table 3. Model estimation results for truck traffic behaviors in Case II

\begin{tabular}{|l|c|c|}
\hline Daily truck demand HGV & 3,521 & PCU \\
\hline Daily truck demand DGV & 8,337 & PCU \\
\hline Total daily truck travel distance & 290,939 & P-kilometer \\
\hline Total daily truck travel time & $4,841.17$ & PCU-hour \\
\hline
\end{tabular}


Fig. 5 presents the flow on the desire link within Case II as the result from GIS map. Result shows that the location condition in intermediate zone is quite far from the terminal location when compare with Case I. This location condition forces all the large vehicles to use outer link road instead causing the distance and traveling time increase as well.

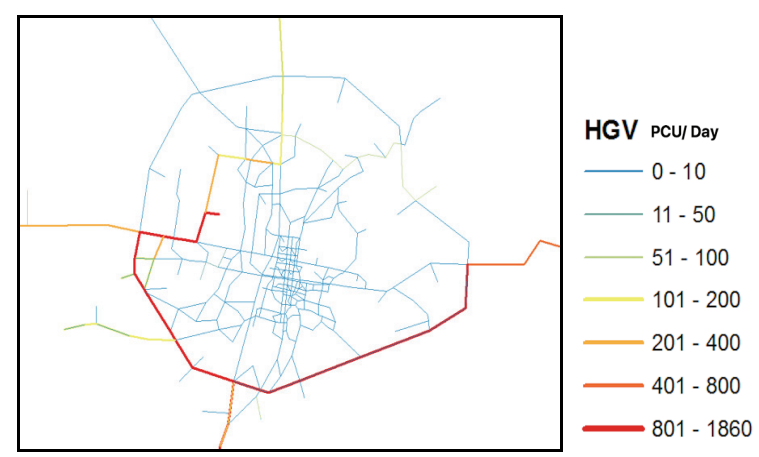

Fig. 5. HGV Traffic flow on links in Case II

Fig. 6, same method is applied on the truck movement so most goods turned to use inside city road and other roads along with intermediate zone. This demonstrates that the road along intermediate zone became significantly red. This indicated the heavy traffic on the link for those delivery trucks.

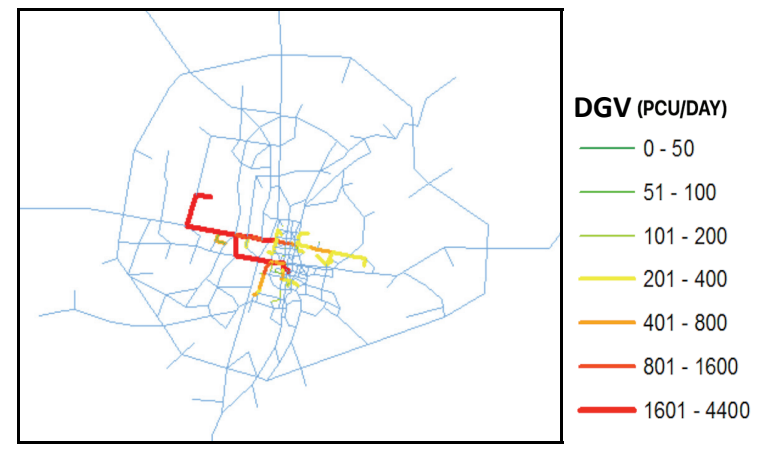

Fig. 6. Delivery Traffic flow on links in Case II

\subsection{Case III Scenario}

For the Case III which the truck terminal is assumed to be located 12 kilometers to the North of the CBD. The result is presented in Table 4. Total truck traveling distances are 148,507 PCU-kilometers, which is about 50 percent less than Case II. However, it is yet greater than Case I by 25 percent. Total traveling distance is 2,625 hours greater than Case I about 22 percent but it is 45 percent better than Case II.

Table 4. Model estimation result for truck traffic behaviors in Case III

\begin{tabular}{|l|c|c|}
\hline Daily truck demand HGV & 3,521 & PCU \\
\hline Daily truck demand DGV & 8,337 & PCU \\
\hline Total daily truck travel distance & 148,507 & P-kilometer \\
\hline Total daily truck travel time & $2,625.74$ & PCU-hour \\
\hline
\end{tabular}

Fig. 7 and Fig. 8 demonstration the traffic flow of large trucks in the Case III. After the road that used for goods delivery is forced to close for truck, the ring road network is showed in the red color and orange color, while the DGVs use the main road from north into CBD.

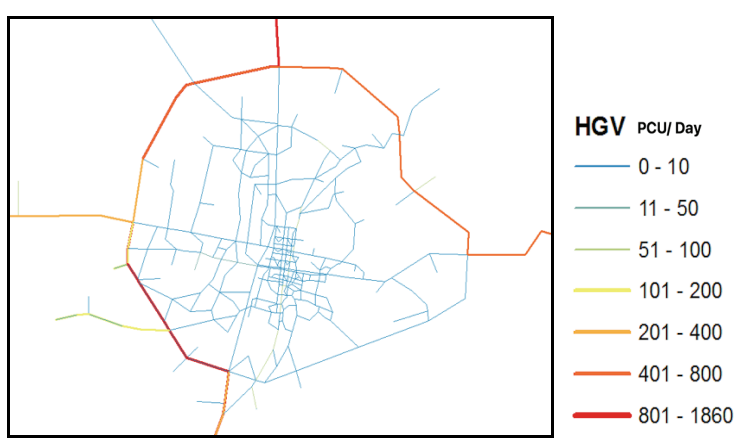

Fig. 7. HGV Traffic flow on links in Case III

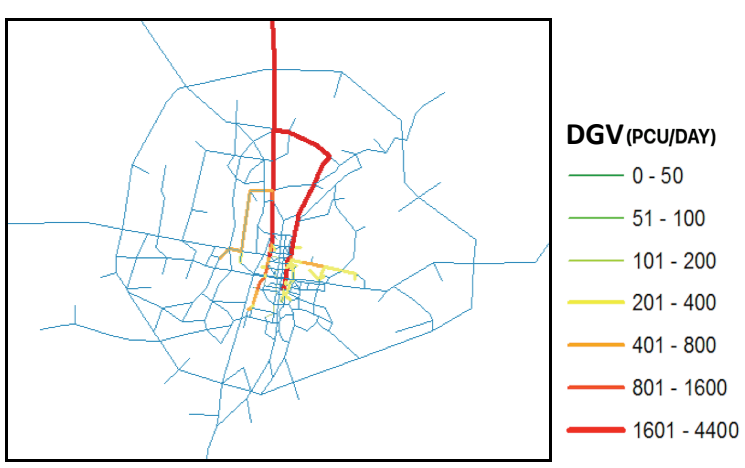

Fig. 8. Delivery Traffic flow on links in Case III

\subsection{Scenarios Comparison}

In this section, the total kilometers and total hours of all three difference cases are evaluated as showed in Fig. 9.

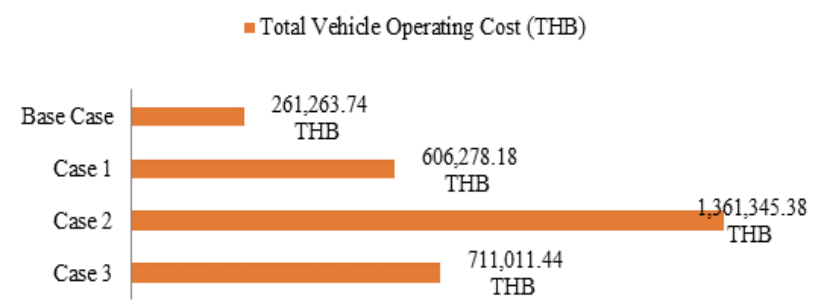

Fig. 9. Total vehicle-operating cost by cases

According to the figure, vehicle operation cost per day for each scenario is compared. Interestingly, Base-Case scenario provides the least cost, with the total cost of approximately 261,000 Thai Baht per day. In contrast, Case II is found to be the worst scenario with the total cost of over 1.36 million Thai Baht, 4 times greater than Base-Case. The truck terminal which gives the best result is Case I. Nevertheless, Case I generates the cost more than 2 times of Base-Case. 


\subsection{Discussion on Results}

After the model results are compared, several interesting discussion can be given.

Firstly, it is found that the location of truck terminal is very crucial. Among the test scenarios, Case I is found to be the best. That is because the location set in the case matches to the actual fright behavior of the city where most of the CBD truck traffics come from or go to the south. For Case II and Case III, all CBD truck traffics are forced to travel along the ring road until reaching the terminal in the other side of the city before switching to small trucks and going back to the CBD Thus, in selecting a truck terminal location, clear understanding on current traffic pattern is necessary.

Secondly, it seems that the idea of implementing truck ban along with an urban truck terminal does not present a good result compared to actual situation. In fact, this finding is expected. In this study, the only direct costs generated by all freight activities in the CBD are taken into account. In the real situation, all trucks go straight from their origin to destination. In all test scenarios on the other hand, all truck traffics are forced to stop by the terminal which are located in suburban area, then all cargo are transshipped to small last-mile delivery trucks and vice versa. This additional activities lead to considerable extra travel distances. Besides, average cost per carrying capacity of delivery trucks, which are basically pick-up trucks, is significantly larger than that of those heavy trucks.

Thirdly, it is also important to note that in this study several costs from activities inside the terminal itself are not taken into account: those costs are, for example, handling, inventory, lost and damage and other terminal overhead costs. If these costs are concerned, it could extend the gap between Base-Case and all three test scenarios.

Although the study findings might lead to a conclusion that the implementation of urban truck terminal along with truck ban policy does not seem to benefit if focusing on direct transport cost. It cannot be said that the scheme is not valuable to the community. There are several benefits in which cannot be measured by the research methodology used in this study: for instance, benefits in term of reducing noise, vibration and air pollutions, traffic accidents, roadside parking issue and traffic obstruction while loading and unloading. These indirect benefits are crucial enough to induce a large number of cities and metropolitans to implement the scheme even if it cannot generate direct benefit in term of direct transport cost.

\section{Conclusion}

This study employs a four-step urban transport model to examine the potential benefits and costs of introducing the truck ban policy along with a development of an urban truck terminal in term of total direct transport cost of the network. Different terminal location scenarios are set to compare with Base-Case scenario. According to the study findings, it is interestingly found that the scheme does not improve or benefit the network in term of direct transport cost. In contrast, the scheme is fond to generate extra cost to the network. Additionally, it is found that the location of truck terminal plays a very important role and need to be matched to the actual behavior.

As discussed earlier, implementation of a truck ban policy along with an urban truck terminal development might not present benefits in term of direct transport cost. Nonetheless, there are several other benefits to the communities. This leaves a room for conducting future work in evaluating direct and indirect benefits of the scheme in other aspects.

\section{Reference}

1. C. Vuchlung \& J. Prabnasak, An Evaluation of the Truck Ban Regulation and Suburban Truck Terminal Implementation Scheme in Regional Cities of Thailand: A Case Study of Khon Kaen City, Thailand., The Sixth International Conference on Transportation and Logistics Proceedings, Taipei (2016)

2. C. Vuchlung, An Evaluation of the Truck Ban Regulation and Suburban Truck Terminal Implementation Scheme in Khon Kaen City of Thailand, King Mongkut's Institute of Technology Ladkrabang, Bangkok (2017)

3. Departement of Land Transport, A Feasibility Study on Regional Truck Terminal Development and Operation in Thailand: the Final Report, Bangkok, Thailand. (2015)

4. M.G. McNally, The Four Step Model. Institute of Transportation Studies, University of California, Irvine, CA. (2007)

5. Expressway Authority of Thailand, A Feasibility Study and Preliminary Design of Urban Expressway Network in Khon Kaen City: the Final Report, Bangkok, Thailand. (2015) 